\title{
The effectiveness of uterine parametrics measured by three-dimensional transvaginal sonography in the diagnosis of uterine cavity distortions
}

\author{
Yan Lin, Min Liu, Suhui He, Minmin Yang, Qiumei Wu \\ Department of Ultrasound, Fujian Provincial Maternity and Child Health Hospital, Fuzhou, China \\ Contributions: (I) Conception and design: Y Lin, M Liu; (II) Administrative support: M Liu, S He; (III) Provision of study materials or patients: M \\ Yang, Y Lin; (IV) Collection and assembly of data: Q Wu, Y Lin; (V) Data analysis and interpretation: Y Lin, M Yang; (VI) Manuscript writing: All \\ authors; (VII) Final approval of manuscript: All authors. \\ Correspondence to: Min Liu. Department of Ultrasound, Fujian Provincial Maternity and Child Health Hospital, Daoshan Road 18, Fuzhou 350001, \\ China. Email: doctor_liumin@163.com.
}

\begin{abstract}
Background: To analyze the application values of quantitative parametrics measured by three-dimensional transvaginal sonography (3D-TVS) in the diagnosis of uterine cavity distortions.

Methods: A retrospective analysis was performed on consecutive 113 patients with septate uteruses, as diagnosed consistently by both 3D-TVS and hysteroscopic surgery, between Jan 2016 and Jan 2018. The intrauterine quantitative parametrics were compared between an infertility group and a miscarriage group. Receiver operating characteristic (ROC) curve analysis and evaluation indicators of diagnostic tests were plotted to analyze the relationships between parametrics and uterine cavity distortions.

Results: There were no significant differences in septum width, septum length, uterine angle, remaining uterine cavity length, and distortion rate between the infertility and miscarriage groups $(\mathrm{P}>0.05)$. The distortion rate was positively correlated with septum length, but showed a negative correlation with uterine angle and remaining uterine cavity length (all $\mathrm{P}<0.05$ ). The area under the curve (AUC) of septum length for predicting a distortion rate higher than $50 \%$ was 0.969 ; with a cutoff value of $2.15 \mathrm{~cm}$, and a diagnostic accuracy of $97.14 \%$, sensitivity was $81.80 \%$ and specificity was $98.90 \%$, respectively. Patients with lower septum length coupled with a higher remaining uterine cavity length had a lower risk of uterine cavity distortion.

Conclusions: Septum length was shown to have the most important effect on uterine cavity distortion of all the uterine parametrics measured by 3D-TVS. Patients with septum lengths higher than $2.15 \mathrm{~cm}$ indicated a distortion rate of more than $50 \%$, and tend to experience adverse pregnancy outcomes.
\end{abstract}

Keywords: Three-dimensional transvaginal sonography (3D-TVS); septate uterus; distortion rate; septum length

Submitted Jul 29, 2020. Accepted for publication Nov 12, 2020.

doi: $10.21037 /$ atm-20-6755

View this article at: http://dx.doi.org/10.21037/atm-20-6755

\section{Introduction}

The septate uterus is the most common type of congenital uterine malformation, and commonly causes infertility or habitual abortion. Generally, surgery is the only effective treatment for patients with clinically symptomatic septate uteri. Therefore, a non-invasive imaging modality is urgently needed to effectively and systematically diagnose and assess features of the septate uterus.

Three-dimensional transvaginal sonography (3D-TVS) is a new ultrasound technique that has been applied in clinical practice because of its high diagnostic accuracy (1). Since 3D-TVS is non-invasive and convenient, it could be employed as an effective imaging modality to screen and diagnose septate uteri $(2,3)$. In recent years, studies have 
increasingly focused on the predictive value and quantitative parametrics of 3D-TVS for adverse pregnancy outcomes of patients with septate uteri. Studies have also reported that uterine cavity deformation was an important parametric affecting adverse pregnancy outcomes (4).

Thus, this present study retrospectively analyzed the effects of various quantitative parametrics of 3D-TVS on the morphology of septate uteruses, in order to figure out the mechanisms of morphological changes in septate uterus patients and provide an objective basis for developing a reasonable clinical treatment regimen.

We present the following article in accordance with the STRAD reporting checklist (available at http://dx.doi. org/10.21037/atm-20-6755).

\section{Methods}

\section{Enrolled patients}

From January 2016 to January 2018, patients who were admitted to the Fujian Provincial Maternity and Child Health Hospital due to infertility or obstetric complications were enrolled in this study. Among them, consecutive 113 cases of septate uterus were diagnosed by 3D-TVS and hysteroscopic surgery, including 10 cases of complete septate uterus, and 103 cases of incomplete septate uterus. The age range was 23 to 35 years, and the mean age was of 28 years. Exclusion criteria included uterine fibroids caused by uterine cavity deformation, myomectomy, and inadequate display of the uterine cavity. All patients underwent 3D-TVS examination 3-5 days after menstruation had finished. Finally, hysteroscopy or surgery was used as the diagnostic gold standard. All procedures performed in this study involving human participants were in accordance with the Declaration of Helsinki (as revised in 2013). The Institutional Review Board of Fujian Cancer Hospital approved the protocols of this present study (No.2016213), and written informed consent was obtained from each participant.

\section{Protocols of 3D-TVS examination}

A GE-E8 color Doppler ultrasound (GE Healthcare, Austria GmbH \& Co OG) diagnostic instrument was used, and the frequency of the 3D-TVS volume probe was 5-9 MHz.

After emptying their bladder, the patient assumed the supine lithotomy position. Firstly, the conventional twodimensional vaginal ultrasound was used for scanning. The contour of the uterus and the pelvic cavity were observed by multiple cuts, and the endometrial morphology was the main observational interest. Secondly, the sagittal section of the uterus was selected. After achieving a clear display of the endometrial image, the $3 \mathrm{D}$ model was activated to reconstruct a $3 \mathrm{D}$ image of the uterine cavity, and the structure and morphology of the uterine cavity were able to be observed from any angle.

Ultrasound findings of the complete septate uterus and incomplete septate uterus were as follows: the coronal plane of the uterus showed a flat, slightly bulging or slightly depressed $(<1 \mathrm{~cm})$ uterus. The uterine cavity of the incomplete septate uterus was "Y" shaped; the complete septate uterus with a double cervical cervix showed the "8" shape; the "V" shape was shown by the complete septate uterus, the separation tube reached the intrauterine mouth, and the single cervical canal (Figure 1).

\section{Measurement index}

On the coronal section of $3 \mathrm{D}$-TVS, the quantitative parametrics of the uterine cavity of the incomplete septate uterus were measured, with the line connecting the 2 vertices of the "Y" shape as the bottom line, and the vertical distance of the septum tip to the bottom line. For the length of the septum (F, in $\mathrm{cm}$ ), the distance from the septum tip to the internal cervix represents the length of the remaining uterine cavity $(\mathrm{C}$, in $\mathrm{cm})$. From this, the uterine cavity deformation rate can be calculated by the $\mathrm{F} /(\mathrm{F}+\mathrm{C})$ formula.

\section{Diagnostic criteria}

The results of hysteroscopic surgery are the gold standard for diagnosis. The criteria for 3D-TVS diagnosis of septate uterus are based on the American Reproductive Society classification criteria (5), as follows: (I) Incomplete septate uterus, there is a septum membrane at the fundus, but it does not extend to the cervix, and the shape of the fundus is slightly convex, or a depression of $<1 \mathrm{~cm}$; (II) complete septate uterus, there is a septum and the diaphragm extends from the fundus to the cervix, completely separating the uterine cavity, and the external contour of the fundus is the same as the incomplete septate uterus.

\section{Statistical analysis}

The statistical analyses of the present study were conducted 

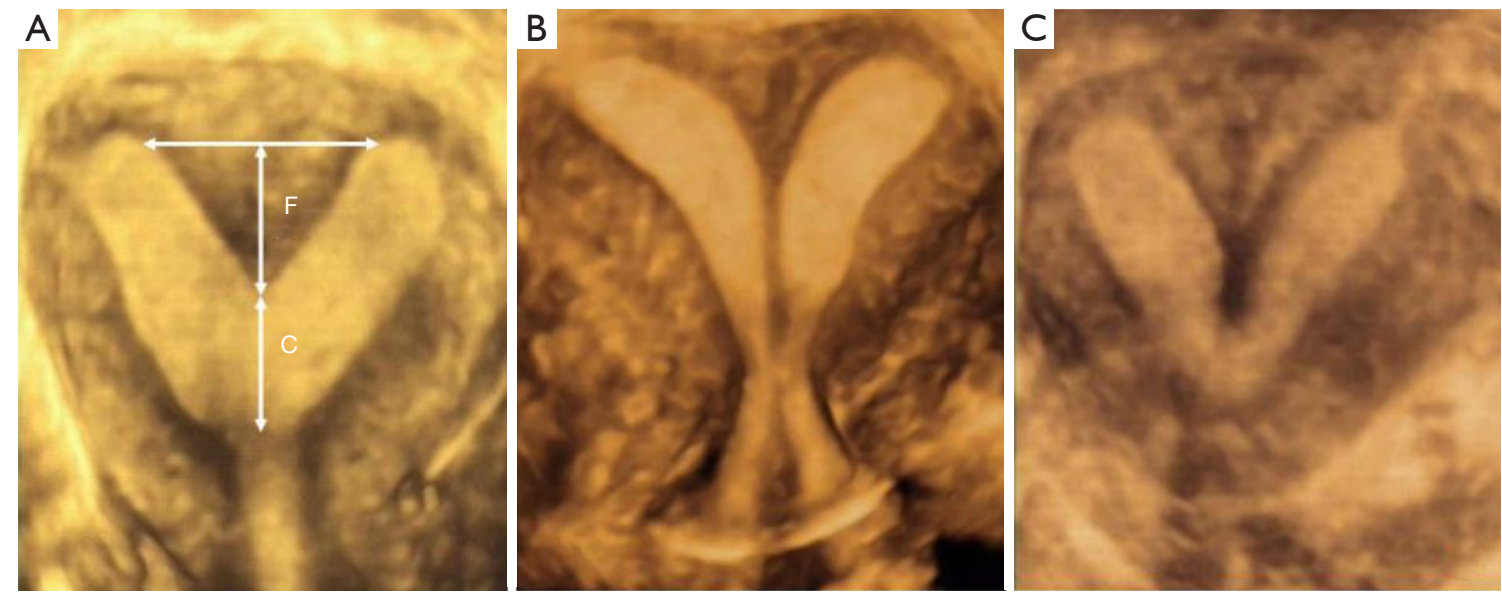

Figure 1 Ultrasound images of the septate uterus. (A) Demonstrates an incompletely septate uterus presenting in a "Y" shape; (B) shows a complete septate uterus in an "8" shape; (C) displays another complete septate uterus in a "V" shape.

Table 1 Comparison of measured uterine parametrics between infertility and abortion patients with incomplete septate uteruses

\begin{tabular}{|c|c|c|c|c|}
\hline Measured parametrics & Infertility group & Abortion group & T-value & $P$ value \\
\hline Septum depth (cm) & $2.82 \pm 0.671$ & $3.02 \pm 0.597$ & -1.431 & 0.156 \\
\hline Septum width (cm) & $1.39 \pm 0.584$ & $1.35 \pm 0.379$ & 0.362 & 0.719 \\
\hline Angle $\left({ }^{\circ}\right)$ & $91.260 \pm 23.51$ & $96.11 \pm 19.85$ & -1.000 & 0.321 \\
\hline Deformation rates of uterine cavity & $0.31 \pm 0.14$ & $0.32 \pm 0.09$ & -0.259 & 0.796 \\
\hline
\end{tabular}

with the SPSS software (version 16.0, IBM Company, Chicago, IL, USA), and the quantitative parametrics of 3D-TVS were expressed. The Student's $t$-test, or one-way analysis of variance (ANOVA) were used to compare the differences in parametrics between different groups. The diagnostic ability of different uterine parametrics were tested by the receiver operating characteristic (ROC) curve analysis. Bivariate correlation analysis was applied using Pearson correlation analysis. Statistical significance was considered at $\mathrm{P}<0.05$.

\section{Results}

\section{Clinical characteristics}

A total of 113 patients were treated for infertility or miscarriage. Preoperative vaginal three-dimensional ultrasound imaging was performed to diagnose the septate uterus. There were 10 completely septate uteruses, accounting for $8.85 \%(10 / 113)$, and 103 cases were incomplete septate uteruses, accounting for $91.15 \%$ $(103 / 113)$. In the complete septate uterus group, there were 3 cases of miscarriage, accounting for $30 \%(3 / 10)$, and there were 7 cases of infertility, accounting for $70 \%(7 / 10)$; in the incomplete septate uterus group, 15 patients were excluded from this study due to the uterine fibroid caused by uterine cavity deformation (4 cases), myomectomy (7 cases), and uterine cavity inadequately displayed (4 cases), respectively; another 88 patients were finally included in this present results, there were 40 cases of miscarriage, accounting for $45.45 \%$ (40/88), and infertility in 48 cases, accounting for $54.55 \%$ (48/88). As shown in Table 1, the study population was divided into infertility and abortion (miscarriage) groups according to the history of adverse pregnancy outcomes in patients with an incomplete septate uterus. The results showed that there was no significant difference in septum width, septum depth, uterine angle, residual uterine cavity length and uterine cavity deformation rate between the infertile group and the abortion group $(\mathrm{P}>0.05)$. 
Table 2 Comparison between deformation rates of the uterine cavity in different measured parametrics of uterus

\begin{tabular}{|c|c|c|c|c|c|c|}
\hline Groups & \multicolumn{6}{|c|}{ Deformation rates of the uterine cavity } \\
\hline Interquartile range of septum depth (cm) & $0.33 \pm 0.11$ & $0.32 \pm 0.12$ & $0.36 \pm 0.17$ & $0.33 \pm 0.11$ & 0.459 & 0.711 \\
\hline Interquartile range of septum width (cm) & $0.25 \pm 0.06$ & $0.29 \pm 0.05$ & $0.35 \pm 0.08$ & $0.50 \pm 0.14$ & 38.136 & 0.000 \\
\hline Interquartile range of angle $\left(^{\circ}\right)$ & $0.46 \pm 0.16$ & $0.35 \pm 0.09$ & $0.30 \pm 0.06$ & $0.24 \pm 0.07$ & 20.680 & 0.000 \\
\hline
\end{tabular}

Table 3 The correlations between different measured parametrics of the uterus and deformation rates of the uterine cavity

\begin{tabular}{lcc}
\hline \multirow{2}{*}{ Parametrics } & \multicolumn{2}{c}{ Deformation rates of the uterine cavity } \\
\cline { 2 - 3 } Septum depth & Correlation coefficient & P value \\
Septum width & 0.862 & 0.000 \\
Angle & 0.073 & 0.470 \\
Remaining uterine cavity length & -0.641 & 0.000 \\
\hline
\end{tabular}

The uterine cavity deformation rate of different measurement parametrics of the uterus

The $25 \%, 50 \%$, and $75 \%$ quantiles of the septum width were $2.51,3.00$, and $3.40 \mathrm{~cm}$, respectively; the $25 \%, 50 \%$, and $75 \%$ quantiles of the septum depth were $1.10,1.29$, and 1.70 $\mathrm{cm}$, respectively; the $25 \%, 50 \%$, and $75 \%$ quantiles of the uterine angle were $76.03,91.50$, and $107.24^{\circ}$, respectively; and the $25 \%, 50 \%$, and $75 \%$ quantiles of the remaining uterine cavity were $2.21,2.80$, and $3.58 \mathrm{~cm}$, respectively. The width, depth, angle, and the interquartile range of the remaining uterine cavity length were divided into 4 groups. The results showed (see Table 2) that the rate of uterine deformation increased with increasing septum depth $(\mathrm{P}<0.05)$. With the increase of the length of the remaining uterine cavity and the angle of the uterus, the rate of uterine deformation became smaller $(\mathrm{P}<0.05)$. However, as the septum width increased, the uterine deformation rate did not change significantly $(\mathrm{P} \geq 0.05)$.

\section{Correlation analysis between different measurement parametrics of the uterus and the rate of uterine cavity deformation}

As shown in Table 3, the results of bivariate correlation analysis showed that the rate of uterine cavity deformation was positively correlated with the depth of uterine septum
$(\mathrm{P}<0.05)$. Uterine cavity deformation was negatively correlated with the length of the remaining uterine cavity $(\mathrm{P}<0.05)$; and there was no correlation with the width of the septum $(\mathrm{P} \geq 0.05)$.

\section{The effect of different septum depths and residual uterine cavity lengths on the rate of uterine cavity deformation}

The septum depth and the $50 \%$ gradation of the remaining uterine cavity length are bound; HF indicates that the septum depth is $>50 \%$ gradation, and LF indicates that the septum depth is lower than $50 \%$ gradation; similarly, the remaining uterine cavity length is divided into $\mathrm{HC}$ and LC. The subjects were reclassified into 4 groups according to the combination of different septum depths and the length of the remaining uterine cavity, namely $\mathrm{LF}+\mathrm{HC}, \mathrm{HF}+$ $\mathrm{HC}, \mathrm{HF}+\mathrm{LC}$, and LF + LC. It can be seen in Table 4 that the $\mathrm{LF}+\mathrm{HC}$ combined uterine cavity deformation rate is the lowest $(0.26 \pm 0.06)$, and the HF + LC uterine cavity deformation rate is the highest $(0.41 \pm 0.13)$.

\section{The value of relevant detection parametrics in determining the uterine deformation rate of patients with incomplete septate uteri}

It is known that the rate of uterine deformation is closely 
Table 4 The deformation rates of the uterine cavity compared between different combinations of the septum depth and the length of remaining uterine cavity

\begin{tabular}{lccccc}
\hline Combinations & LF $+\mathrm{HC}$ & $\mathrm{HF}+\mathrm{HC}$ & $\mathrm{HF}+\mathrm{LC}$ & $\mathrm{LF}+\mathrm{LC}$ & $\mathrm{F}$-value \\
\hline Cases $(\mathrm{N})$ & 99 & 95 & 101 & 105 & $\mathrm{P}$ value \\
Septum deformation rate & $0.26 \pm 0.06$ & $0.34 \pm 0.13$ & $0.41 \pm 0.13$ & $0.33 \pm 0.13$ & 27.784 \\
\hline
\end{tabular}

HF, the septum depth $>50 \%$ gradation; LF, the septum depth $<50 \%$ grading; HC, the remaining uterine cavity length $>50 \%$ gradation; LC, the remaining uterine cavity length $<50 \%$ gradation.

Table 5 ROC curve analysis of different uterine parametrics in the diagnosis of uterine deformation rates

\begin{tabular}{llc}
\hline Parametrics & AUC & $95 \% \mathrm{Cl}$ \\
\hline Septum depth & 0.969 & $0.000-1.000$ \\
Septum width & 0.524 & $0.344-0.704$ \\
Angle & 0.092 & $0.004-0.180$ \\
Remaining uterine cavity length & 0.069 & $0.016-0.122$ \\
\hline
\end{tabular}

ROC, receiver operating characteristic.

Table 6 Diagnostic ability analysis of uterine deformation rates with different mediastinum depth points

\begin{tabular}{|c|c|c|c|c|c|c|c|c|}
\hline $\begin{array}{l}\text { Septum depth } \\
\text { threshold }(\mathrm{cm})\end{array}$ & Sensitivity (\%) & Specificity \%) & PPV (\%) & NPV (\%) & Accuracy (\%) & $\begin{array}{c}\text { Missed diagnosis } \\
\text { rate }(\%)\end{array}$ & $\begin{array}{c}\text { Misdiagnosis } \\
\text { rate }(\%)\end{array}$ & $\begin{array}{c}\text { Youden index } \\
(\%)\end{array}$ \\
\hline 1.7 & 100 & 70.10 & 27.50 & 100 & 72.90 & 0 & 29.9 & 70.10 \\
\hline 2.15 & 81.80 & 98.90 & 89.50 & 97.93 & 97.14 & 18.20 & 1.10 & 80.70 \\
\hline 2.25 & 63.60 & 98.90 & 86.89 & 95.95 & 95.27 & 36.4 & 1.10 & 62.50 \\
\hline
\end{tabular}

PPV, positive predictive value; NPV, negative predictive value.

related to adverse pregnancy outcomes. The literature reports that the uterine deformation rate $>51 \%$ can be used as the critical value for the occurrence of adverse pregnancy (4). Therefore, the present study defined the uterine deformation rate $>50 \%$ as the risk signal value, and analyzed the predictive effect of each uterus parametric on the rate of uterine deformation. As shown in Table 5, in all the measured uterine indexes, the septum depth had the highest diagnostic value for the uterine deformation rate, and the area under the curve (AUC) for the ROC was 0.969 . The other indicators have no diagnostic value, and the AUC was $<0.6$.

From the ROC curve of the septum depth, the diagnostic value of the following boundary value for the uterine deformation rate was analyzed. The highest diagnostic accuracy rate was $2.15 \mathrm{~cm}$ for the septum depth, the highest diagnostic accuracy was $97.14 \%$, and the most sensitive and specific was close to $81.80 \%$ and $98.90 \%$ respectively, as shown in Table 6.

\section{Discussion}

The septate uterus is the most common congenital uterine malformation, which is caused by the incomplete degeneration of the septum after the confluence of the bilateral renal tube in the development of the embryo. When the septum divides the entire uterine cavity, a complete septate uterus is formed, and when the septum is partially degenerated, an incomplete septate uterus is formed. Under conventional circumstances, the septate uterus is more likely to cause miscarriage, premature delivery or abnormal fetal position, postpartum placental adhesion or placenta retention, and other adverse outcomes, all of which commonly contribute to infertility. Previous 
studies have shown that the incidence of early abortion in pregnant females with incomplete septate uteruses was of $42 \%$, which is significantly higher than that of normal uterine pregnancy (12\%) (6). Early and accurate diagnosis of the septate uterus can guide early adoption of clinical measures to improve the rate of conception and avoid miscarriage, premature birth and so on. Therefore, the high diagnostic accuracy of high-resolution and non-invasive imaging examinations for the screening and diagnosis of the septate uterus is particularly considerable.

Comparing to the conventional 2D-TVS, 3D-TVS can display three-dimensional images, with higher positioning accuracy and good visual real-time performance. With 3D-TVS the conventional section of uterus, as well as the coronary and axial section can be obtained simultaneously, which provides complete acoustic information about the internal and external structures of uterus. 3D-TVS can also observed the wall of uterus, endometrial and intrauterine lesions from different directions simultaneously, especially for detecting the micro-lesions and accurately positioning their site and scope, which can provide abundant imaging information for subsequent hysteroscopy biopsy or surgery. Thus, the 3D-TVS is highly accurate for the observation and diagnosis of septate uterus (1). In recent years, the clinical value of 3D-TVS in diagnosing the septate uterus, coupled with its quantitative parametrics, has gained increasing attention for the ability to effectively evaluate and predict adverse pregnancy outcomes.

Previous studies have revealed the rate of uterine cavity deformation as a good indicator of whether abortion will occur in patients with an incomplete septate uterus, and the optimal threshold was 0.51 (4). This study retrospectively analyzed patients with septate uteruses with histories of adverse pregnancy outcomes (habitual abortion and primary infertility) that were eventually diagnosed and confirmed by hysteroscopy. It was found that in patients with incomplete septate uteri, there was no significant difference in the quantitative parametrics of $3 \mathrm{D}$-TVS between those of abortion and primary infertility. Our study compared patients with incomplete septate uteruses who had a history of adverse pregnancy with those without a history of adverse pregnancy, the results demonstrated that the former has a higher rate of uterine deformation and a longer septum depth (not shown in this paper). These comparative findings suggested that the measured parametrics of 3D-TVS could predict the incidence of adverse pregnancy outcomes by judging the uterine morphological abnormalities., nevertheless, the uterine morphological abnormalities diagnosed by 3D-TVS are just the factors that will cause the abortion and infertility, it did not help to distinguish between miscarriage and primary infertility.

The occurrence of the septate uterus changes the morphology of the uterine cavity and interferes with the normal implantation and development of the embryo. The characteristic of the septate uterus is that the septum tissue is relatively diminished, the fibrous tissue is increased, and the intima covering the septum is often stunted; the responsiveness to hormones is also higher and poorly regulated $(7,8)$. Therefore, uterine cavity deformation consistently and directly affects pregnancy outcomes. To this end, the present study explored the effects of various $3 \mathrm{D}$-TVS quantitative parametrics on the rate uterine deformation, in order to discern the major risk factors (i.e., Arch uterus, Biangular uterus, and Double uterus, etc.) for predicting adverse pregnancy outcomes. Firstly, according to the numerical range of each parametric measurement, patients were divided into 4 groups of different grades with 4-point separation. It was also revealed that the uterine deformation rate was positively correlated with septum depth; however, with the remaining uterine length, the angle of uterus deformation gradually decreased. Further correlation analysis found that the rate of uterine cavity deformation was negatively correlated with the angle of the uterus and the length of the remaining uterine cavity. In different combinations of uterine lumen lengths, the probability of uterine cavity deformation is lowest when septum length was low and residual uterine length was high. Therefore, we speculate that the above 3 parametrics have an important influence on uterine morphology. The ROC curve analysis also found that the diagnostic performance of the 3D-TVS quantitative parametrics for the rate of uterine deformation was significant, and septum depth had the highest diagnostic value for uterine deformation rate in the diagnosis of septate uterus, with a high AUC value of 0.945 . However, the ROC curve analysis showed that the other indicators had no significant diagnostic value. Thus, in the above-mentioned measurement index, the septum depth is the most important parametric affecting the morphology of the uterus.

In conclusion, among the quantitative parametrics of the uterine cavity measured by $3 \mathrm{D}-\mathrm{TVS}$, the septum depth, the remaining uterine cavity length, and the uterine angle were the main indicators affecting the morphology of the uterus. The septum depth was the most significant parametric that indirectly reflected the deformation of the uterine cavity. Septum depth can further provide an objective reference 
indicator to predict the occurrence of adverse pregnancy outcomes, and also assist in the development of a reasonable treatment regimen.

\section{Acknowledgments}

Funding: This study was supported by the Medical Union Project of Fujian Province Natural Science Foundation (Grant No. 2019J01513).

\section{Footnote}

Reporting Checklist: The authors have completed the STRAD reporting checklist. Available at http://dx.doi. org/10.21037/atm-20-6755

Data Sharing Statement: Available at http://dx.doi. org/10.21037/atm-20-6755

Conflicts of Interest: All authors have completed the ICMJE uniform disclosure form (available at http://dx.doi. org/10.21037/atm-20-6755). The authors have no conflicts of interest to declare.

Ethical Statement: The authors are accountable for all aspects of the work in ensuring that questions related to the accuracy or integrity of any part of the work are appropriately investigated and resolved. All procedures performed in this study involving human participants were in accordance with the Declaration of Helsinki (as revised in 2013). The Institutional Review Board of Fujian Cancer Hospital approved the protocols of this present study (No.2016213), and written informed consent was obtained from each participant.

Open Access Statement: This is an Open Access article distributed in accordance with the Creative Commons Attribution-NonCommercial-NoDerivs 4.0 International
License (CC BY-NC-ND 4.0), which permits the noncommercial replication and distribution of the article with the strict proviso that no changes or edits are made and the original work is properly cited (including links to both the formal publication through the relevant DOI and the license). See: https://creativecommons.org/licenses/by-nc-nd/4.0/.

\section{References}

1. Faivre E, Fernandez H, Deffieux X, et al. Accuracy of three-dimensional ultrasonography in differential diagnosis of septate and bicornuate uterus compared with office hysteroscopy and pelvic magnetic resonance imaging. J Minim Invasive Gynecol 2012;19:101-6.

2. Reid S, Nadim B, Bignardi T, L et al. Association between three-dimensional transvaginal sonographic markers and outcome of pregnancy of unknown location: a pilot study. Ultrasound Obstet Gynecol 2016;48:650-5.

3. Bermejo C, Martínez-Ten P, Ruíz-López L, et al. Classification of Uterine Anomalies by 3-Dimensional Ultrasonography Using ESHRE/ESGE Criteria: Interobserver Variability. Reprod Sci 2018;25:740-7.

4. Chen Y, Cai A, Yang Q, et al. Transvaginal 3-D Ultrasonic Sonohysterography for Clinical Evaluation of Septate Uterus. Chinese J Ultrasound Med 2006;22:128-30.

5. ASRM. Uterine septum: a guideline. Fertil Steril 2016; 106: 530-540.

6. Woelfer B, Salim R, Banerjee S, et al. Reproductive outcomes in women with congenital uterine anomalies detected by three-dimensional ultrasound screening. Obstet Gynecol 2001;98:1099-103.

7. Vaz SA, Dotters-Katz SK, Kuller JA. Diagnosis and Management of Congenital Uterine Anomalies in Pregnancy. Obstet Gynecol Surv 2017;72:194-201.

8. Biler A, Akdemir A, Peker N, et al. A Rare Uterine Malformation: Asymmetric Septate Uterus. J Minim Invasive Gynecol 2018;25:28-9.

(English Language Editor: J. Jones)
Cite this article as: Lin Y, Liu M, He S, Yang M, Wu Q. The effectiveness of uterine parametrics measured by threedimensional transvaginal sonography in the diagnosis of uterine cavity distortions. Ann Transl Med 2020;8(22):1486. doi: 10.21037/atm-20-6755 